\title{
PAREIDOLIA: CHARACTERISING FACIAL ANTHROPOMORPHISM AND ITS IMPLICATIONS FOR PRODUCT DESIGN
}

\author{
Authors \\ Dr. Andrew Wodehouse (corresponding author), Ross Brisco, Edward Broussard and Professor \\ Alex Duffy \\ Department of Design, Manufacture and Engineering Management \\ University of Strathclyde, James Weir Building, 75 Montrose Street, Glasgow, G1 1XJ. \\ Tel. +44 (0)141-548 2091 \\ Fax. +44 (0)141-552 7986 \\ Email. andrew.wodehouse@strath.ac.uk
}

\begin{abstract}
This work highlights the phenomenon of pareidolia - the tendency to see faces in the environment, buildings and objects that surround us - and establishes its relevance for design contexts. In reviewing literature on anthropomorphism and the use of faces in design embodiment, we have shown that it is a compelling and prevalent facet of how we interpret products. By surveying 2,309 images from across the internet, we provide the first systematic investigation of product types and face characteristics (size, composition, emotion) that are manifest in this phenomenon. The most common instances were shown to be in medium-sized products, where part of the product was interpreted as a face, and that conveyed a happy emotion. The effects of culture and self-congruence are identified as important aspects of our interpretation of facial emotion. It is concluded that the fundamental geometric elements of products should be considered with respect to facial morphology, whether it be the intention to utilise its effects or not, and set out case for more quantified guidelines on the use of pareidolia and anthropomorphism in design.
\end{abstract}

\section{Keywords}

Pareidolia; anthropomorphism; aesthetics; facial representation; emotion.

\section{Introduction}

This work sets out to explore the phenomenon of pareidolia - the tendency to see faces in the environment, buildings and objects that surround us - with a focus on the implications for designers when considering the geometry, styling and configuration of products. By reviewing the places and products in which the phenomena occurs and then assessing the emotions conveyed, we provide a series of insights that should be considered when developing an industrial design for a product that may have elements that could be considered similar to a face. Pareidolia is a phenomenon that can easily happen accidentally, in which case there can 
be unexpected or undesirable reaction in users, or it can be used deliberate to invoke a particular emotion or response. We are therefore highlighting its characteristics and impact so that designers can assess whether it is relevant to their design detailing, and to incorporate appropriately or consciously eschew it as appropriate.

While pareidolia is specifically concerned with faces, anthropomorphism is the broader attribution of human characteristics to objects, animals or events. It is therefore useful to understand is history and emergence of the broader phenomenon before exploring the practical implications of pareidolia. The earliest known writings on anthropomorphism are by Xenophanes (c.570-c.475BC) the Greek philosopher who satirized the projection of human forms to the gods:

\section{'Ethiopians say that their gods are snub-nosed and black}

Thracians that they are pale and red-haired' (Lesher 2001)

The tendency to explain natural phenomena in human terms is highly visible across many cultures and civilizations, from the prehistoric anthropomorphic figurines thought to represent a Mother Goddess (Ucko 1962) to the modern Western convention of naming hurricanes. It is only in more recent times with the rise of monotheistic religions such as Islam, Christianity and Judaism that the assignation of human characteristics to deities has been discouraged. Further, the spirit of philosophical and scientific enquiry has viewed anthropomorphism with suspicion on the basis that it is an impediment to a clear understanding of the world around us.

While anthropomorphism can assign human characteristics to phenomena, it also operates powerfully in visual terms. As social animals we are highly attuned to human shapes, expressions and movement. Empathy is such a fundamental part of human experience that specific 'mirror neurons' have been identified in helping us understand the world from others' point of view (Frazzetto 2013), and theories of embodied cognition that account for the process of emotional understanding (Winkielman et al. 2008). The evolutionary perspective goes some way to explaining why we talk of being 'hard wired' to remember faces rather than names - in terms of human development, the need to recognise and remember individuals came long before the capacity for speech (McGilchrist 2010). Indeed, the perception of faces and facial representation is regarded as one of the most highly developed human skills (Haxby et al. 2000). Morton and Johnson (1991) showed that infants prefer looking at faces rather than any other objects from a young age, and over a lifespan most people spend more time looking at a facial representation either in person or through some type of media than any other object (Haxby et al. 2000).

This tendency to seek and recognise facial configurations and expressions extends beyond communication with other people to the objects that surround us. The term pareidolia is defined as "the imagined perception of a pattern or meaning where it does not actually exist..." derived from the Ancient Greek (para, "amiss, wrong" + eidōlon, "image"). This detection of a Gestalt from seemingly vague or random stimulus has been termed as the "aesthetic aha!" (Leder and Carbon 2005; Muth and Carbon 2013). While the phenomenon can be traced to our distant past, access to digital cameras to conveniently capture images and the internet to share and discuss these has led to a much higher awareness and discussion of its power. This is reflected in the marked increase in use of the term and the rise in websites, blogs and Twitter feeds devoted to identifying instances. While there is an element of frivolity to spotting these, the satisfaction one feels when the face unexpectedly reveals itself highlights our desire to make sense of a complex world (Figure 1). 
The aim of this research is to understand the role and impact of this phenomenon in the context of industrial design. In reviewing the instances of pareidolia commonly posted to the internet, we noticed a high prevalence in relation to products. Given the potential implications for a designer, we have therefore considered the following research questions:

- How prevalent is pareidolia in relation to product design?

- What are the relevant product characteristics in instances where pareidolia occurs?

- What emotions are most commonly induced and how can these be harnessed by designers?

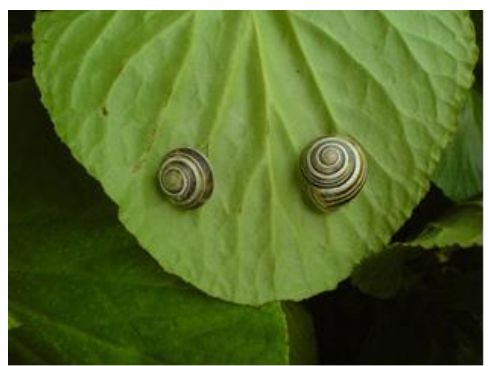

Nature

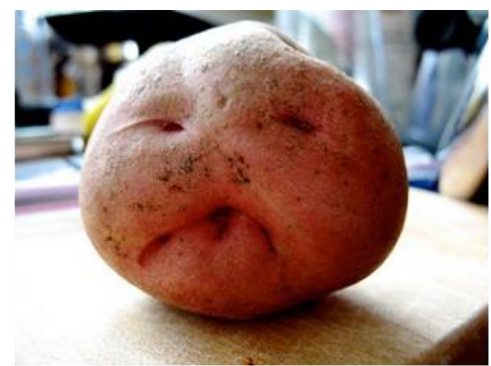

Food

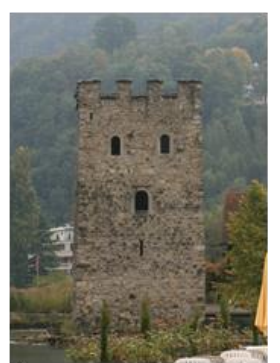

Architecture

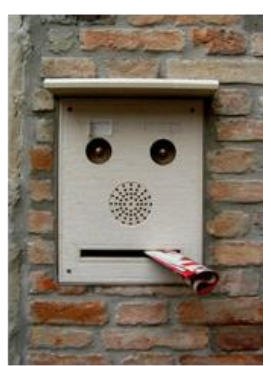

Products

Figure 1: Tendency to see faces in nature, food, architecture and products

\section{Pareidolia in design}

Given the propensity for anthropomorphism, and pareidolia specifically, to manifest itself either by accident or intentionally, it is important for designers to understand how it can be used to enhance rather than compromise design configurations. For example, DiSalvo and Gemperle (2003) suggest that anthropomorphic forms can be utilised in a number of settings: where it has become convention for a category of product; to help make new technologies more understandable; to reflect a product's functional attributes; or to project human values. Similarly, Mensvoort and Grievnik (2011) propose a set of eleven 'rules' addressing issues such as cultural differences, the tendency to simplify complex products, aligning with human ethics and the importance of purpose when considering the use of anthropomorphism. Empirical studies with existing products have outlined aspects such as personality (Mugge et al. 2009) and familiarity (Faerber and Carbon 2012) in determining user reaction.

Product design is increasingly concerned with how we can make products more pleasurable to use, more durable and perform better by being responsive to tacit, emotional needs (Chapman 2005; Norman 2004). Anthropomorphism therefore clearly has a role in product aesthetics to improve human response and thus the performance of products ( $\mathrm{Wu}$ and Chang 2007; Hoegg and Alba 2011). Our predisposition to finding human shapes and patterns (Arnheim 1969) points to a deep-seated predilection for pareidolia. Both rational and emotional theories have been presented to explain this. The rational position is that it is a way to understand the world by referring to something we know intimately i.e. ourselves, assigning values or ethics (Nam and Kim 2011) or to exert authority over our surroundings (Claxton and Murray 1994). On the other hand, suggested emotional drivers include a desire to make the world less threatening, and the need for social contact or interaction (Epley et al. 2007). What is implicit in both these perspectives is the prominent role of the self, our role in the world (selfcongruence) and how we react to the objects that surround us (perception). 
Previous studies have shown that consumers prefer products with a symbolic meaning that aligns with their own self-image (Govers and Schoormans 2005). Self-congruity theory as dictates that consumers evaluate product-user image of a product against their own self-concept (Quester et al. 2000; Sirgy 1982). Its key tenet is that consumers are drawn to a product only if they feel it is consistent with, or has the ability to enhance, their perception of themselves or who they want to be (Quester et al. 2000; Ross 1971). Research in the emotional design domain (Govers et al. 2003) has shown the difficulties of portraying product character through design in a way that consumers understand and interpret correctly. Also, as with all product symbolisation issues self-concept is closely related to cultural differences (Hall and Gay 1996).

Facial expressions offer the most effective and common channel for expression of emotion (El Kaliouby et al. 2003; Baron-Cohen et al. 2001). Only a very short period of time (below $40 \mathrm{~ms}$ ) is needed to recognise a face properly (Carbon 2011) and even in cases of prosopagnosia ("face blindness"), it is possible to efficiently determine attractiveness (Carbon et al. 2010). Small variations in expression can affect our perception (Mitteroecker et al. 2013), and even a photograph has the ability to influence the emotion of the person viewing it, giving them an instantaneous insight into the person's emotional state (Ekman 1980; Willis and Todorov 2006). Studies have begun to develop frameworks for the interrogation of geometrical differences in product categories such as smartphones (Ranscombe et al. 2012) and eyeglasses (Lo and Chu 2012) that work back from existing examples to understand how features can affect our reactions. Similarly, the presentation of symbolic elements to experimental participants has been used to assess emotional response and the transferability to product design (Desmet 2003; Govers and Schoormans 2005; Quester et al. 2000).

In Figure 2 we illustrate how the phenomenon of pareidolia can emerge in product configurations and its role in product anthropomorphism. The lathe on the left is a non-facial configuration of geometrical components that have elements of symmetry and repetition. We regard this as an abstract product layout that invokes little or no interpretation of human forms. The car dashboard in the centre has strong circular and horizontal elements that can be construed as facial features, though it may not be immediately obvious. This accidental or unclear emergence of a face we denote as accidential paredolia and if not recognised by the designer can cause unexpected reactions in users. The facia on the right is clearly similar to laughing human face through the use of the crescent eyes and upturned mouth. This invocation of a human expression is the deliberate use of pareidolia and product anthropomorphism imbuing the product with human characteristics.

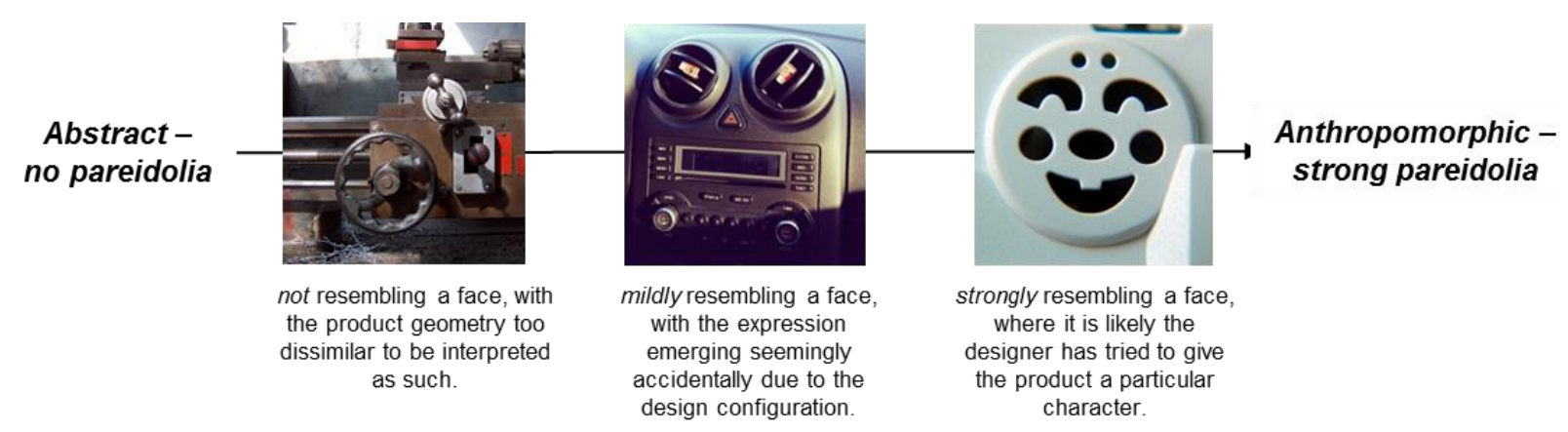

Figure 2: Examples of non-, mild and strong facial resemblance using basic geometric shapes 


\section{Previous utilisation of facial phenomena}

We wish to describe how facial perception can be used as a tool to help designers when forming key product geometries across categories or sectors. Two areas that have already received particular attention, however, are automotive styling and robotics. We review each in turn to provide a better understanding of how anthropomorphism has been used in the past, and how it will be in the future.

\subsection{Automotive elevations}

Cars are a dominant feature of our society. They are the largest items of machinery with which we commonly interact, dictating our civil infrastructure and tempo of life. Requiring significant financial investment and relied upon for both the necessities of pleasures of movement, they often inspire devotion in the form of serial brand loyalty, garage customisation and pet names. As a well-established product category, the car is essentially static in its functionality - moving us from one place to another on four wheels. While manufacturers continue to incrementally improve performance indicators such as fuel efficiency, they rely heavily on 'delighters' to differentiate their models from the competition (Chitturi et al. 2007). These are the emotional and less tacit elements that have led to the automotive sector forming an important industry in developing ideas on aesthetics (bodywork styling), interaction design (dashboard control) and even sound design (the sound of doors closing).

The frontal elevation of a car has an obvious facial connotation and has attracted a number of studies on how variations in proportions and variations can affect user reaction. It has been suggested that in the 'faces' of cars we assign characteristics in the same way as we do to human configurations and expressions (Windhager et al. 2008). Miesler et al. (2011) adopt an evolutionary perspective to conclude that a 'baby schema' of proportionately large eyes (in this case headlights) encourages a perception of 'cuteness'. This aligns with subsequent findings from Purucker et al. (2014) identifying cute and threatening designs using evolutionary theory. Aggarwal and McGill (2007) highlight the importance of aligning the features of the car with consumer expectations of the car's 'persona'. While in broad terms it has been shown that consumers prefer smiling car grills with aggressive eyes because this combination of features provides the most pleasure and arousal (Landwehr et al. 2011), recent studies have suggested that anthropometric features (albeit behavioural rather than visual) can encourage trust in autonomous vehicles (Waytz et al. 2014), and that using 'threatening' expressions can increase initial attention in users (Purucker et al. 2013).

From the volume and similarity of work conducted in this area, it seems likely that we can imbue a product with particular characteristics depending on how strong the facial morphology is, and the expression it subsequently takes. These are important considerations not just for cars where the facial analogy is somewhat inevitable, but for any design situation where a face could be construed.

\subsection{Robot faces}

Robotics is a particularly emotive category of product, as their purpose is often to emulate or replace human interactions. As robots become increasingly prevalent in our day-to-day lives, whether they should try to imitate human forms becomes a pressing issue. It has been suggested that people empathise more strongly with human looking than mechanical robots (Riek et al. 2009). A widely recognised phenomenon, however, is the 'uncanny valley' (Mori 1970) whereby robots that are realistic but noticeably artificial take on a disturbing quality that repels the user. In attempting to quantify anthropomorphic realism in robots, Bartneck et al. (2009) 
use the semantic differentials of fake/ natural, machine-like/ human-like, unconscious/ conscious, artificial/ lifelike, and moving rigidly/ moving elegantly.

Other work has explored the different modes of expression and communication to convey anthropomorphism. Whether the robot is highly realistic or not, facial expressions have been shown to play an important role in user perception (Canamero and Fredslund 2000). Ortony and Turner (1990) found that users were adept at discerning the 'basic emotions' in a rudimentary humanoid robot - with the exception of fear, which was misinterpreted as anxiety, sadness or surprise. Similarly, Hegel et al. (2006) concluded that a robot which utilised facial expressions when reading text was perceived as having a better understanding of emotional context. Other tacit forms of communication such as body language can help build a rapport with users - it has been reported that people respond well to gestures accompanying speech in robots, even when they are incongruous (Salem et al. 2013). Indeed, the ability to establish a (real or imagined) social rapport is critical to successful anthropomorphism in robots: in their 'three-factor' theory, Epley et al. (2007) emphasise the motivation of people to communicate, particularly when lacking social connection with other humans.

Based on a 'Sliding Scales of Anthropomorphism' (2014) and established terminologies (Riek et al. 2009), we have developed a set of categories for different robot types (functional, assistant, humanoid, android, uncanny, cyborg) and the facial phenomena (abstract, pareidolia, anthropomorphism, uncanny valley, realistic) in relation to them. While terms such as android and cyborg have been used interchangeably in the past, the definitions in Figure 3 provide a framework to understand how the functionality and context of the robot may contribute to the need and nature of the realism in robotic faces. We can anticipate that much of the design in robots of the coming years will be in the categories of assistant through to android as the technology to convincingly emulate humans simply does not exist. In these circumstances, it is more practical to utilise features or characteristics that can elicit particular characteristics rather than strive for something that is obviously imperfect. If pareidolia is considered the accidental emergence of faces, it is between the categories of functional and assistant robots that we should be wary of its occurrence. Indeed, moving beyond the human and employing zoomorphic analogies such as dogs or spiders may be appropriate to inspire confidence in assistant robots. The deliberate utilisation of human characteristics anthropomorphism - becomes relevant at the humanoid and android level of realism, where particular characteristics can be conveyed through the styling and detailing. For example, high levels of geometric detail may suggest intelligence or functional sophistication, whereas deliberately rounded features and use of proportion may imbue humanoids with cuteness. In moving towards cyborg robots, we should be aware of the discomfort caused by the uncanny valley. Even when a static robot is convincing, if it is unnatural in its movement or expressions can similarly invoke revulsion. Until a highly convincing robot face can be constructed, it may be better for designers to deliberately invoke more abstract android elements to instil character to their robot faces. 


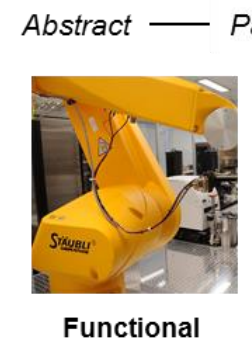

Robot built for a particular function, often industrial.

Generally

anonymous and

lacking any

anthropomorphic

characteristics.
Assistant

Moving away from humanoid forms, often takes on animal or pet characteristics (zoomorphism) to act
as a 'buddy' for particular tasks.
Anthropomorphism

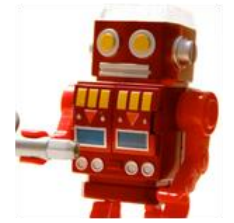

Humanoid

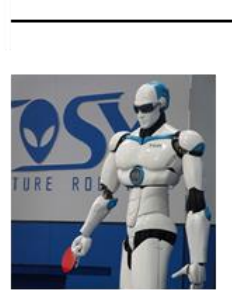

Android

Tends to be made up of simple blocks or cylinders to give the broad impression of a (usually deferential) humanoid.
Uncanny valley

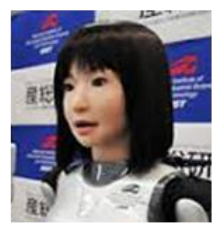

Uncanny

Robots made lifelike but falling into the 'uncanny valley' of visual discomfort where, either through aesthetics or movement, are identified as artificial.

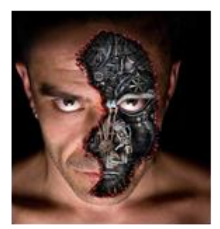

Cyborg

A part-human partmechanical organism that takes on a human appearance.

\section{Figure 3: Categories of robot and facial phenomena, after 'The Sliding Scale of}

Anthropomorphism' (2014) and Riek et al. (2009)

\section{Data collection and results}

We decided to explore the broad patterns of emergence of pareidolia by examining the evergrowing number of images that have been uploaded across the internet. In each of these instances, someone has unexpectedly seen a face and felt compelled to photograph and upload an image to a pareidolia database. In evaluating the nature and composition of images uploaded, we can interpret the places and types of items that are memorable enough for people to upload. Although we cannot directly ask for feedback, the fact they have been uploaded means that we can make inferences from the body of images. A range of sources were identified as set out in Table 1. The majority have come from the blog "Faces in Places", later reprinted as a book (Smith 2010) and courtesy of which many of the images used in this paper appear, but a total of five different sources were utilised.

\begin{tabular}{|l|l|l|}
\hline Name & Address & $\begin{array}{l}\text { No. of images } \\
\text { downloaded }\end{array}$ \\
\hline $\begin{array}{l}\text { Buildings That Look Like } \\
\text { Faces }\end{array}$ & http://buildingsthatlooklikefaces.tumblr.com & 18 \\
\hline Faces In Places & http://facesinplaces.blogspot.co.uk & 2,131 \\
\hline Face In A Place & $\begin{array}{l}\text { http://www.faceinaplace.com/category/faces } \\
\text {-in-buildings }\end{array}$ & 50 \\
\hline $\begin{array}{l}\text { Twisted Sifter - 50 Faces } \\
\text { in Everyday Objects }\end{array}$ & $\begin{array}{l}\text { http://twistedsifter.com/2013/05/50-faces-in- } \\
\text { everyday-places }\end{array}$ & 50 \\
\hline Pareidolia & https://www.reddit.com/r/Pareidolia & 60 \\
\hline
\end{tabular}

\section{Table 1: Sources of images with description and number sourced from each}

The images from across the sources were downloaded in August 2015 using HTTrack 3.4821 Website Copier. This resulted in a folder with 2,309 images. A check for duplicates was then carried out using Dupeguru 2.10.1, which identified only three instances, and a manual check identified a further 25 duplications. The remaining 2,281 images were imported into Adobe Photoshop Lightroom CC 2015 for categorisation and organisation. Categorisation was carried out by one of the authors and reviewed by another. The analysis consisted of a total of six 
different 'tags' to identify the product and face that has been noticed. The tags and the order in which they were applied is listed in Table 2.

\begin{tabular}{|l|l|l|}
\hline Order & Tag & Example tags \\
\hline 1 & Type & Nature, Food, Architecture or Product \\
\hline 2 & Category & Gadget, Bedroom, Furniture etc \\
\hline 3 & Size & $\begin{array}{l}\text { Small (handheld), Medium (easily portable) or Large (not easily } \\
\text { portable) }\end{array}$ \\
\hline 4 & Composition & Part of a product, Full Product or Multiple Products \\
\hline 5 & Contrast & High or Low \\
\hline 6 & Emotion & Happy, Sad, Fear, Anger, Surprise, Disgust, Contempt/Neutral \\
\hline
\end{tabular}

Table 2: Categorisation and organisation of images

The images were firstly tagged with a high level nature, food, architecture or product type. This revealed that $62 \%$ of the images were product related and confirmed our perception that products make up the majority of noticed images. From this point onwards the authors focused on the product category only, with the five remained tags used to better characterise the instances of pareidolia in this context. A category tag, adapted from the online shopping website Amazon.co.uk, was used to assign product subsets. This revealed that technical components such as engines and machinery made up $18 \%$ of the total images closely followed by gadgets at $16 \%$. To describe the product context of the faces in more detail, a number of further details were noted: the size of the overall product, the composition of the face as part of the product, and the contrast that the features of the face had with the product background. No useful information was derived from the contrast assessment, so it does not appear in our further analysis.

The emotion of the faces were asserted by reviewing the 'facial features' in the image and comparing it to a human facial expression as described in Ekman (2001), with picture references. While this categorisation was subjective to the reviewing author, it was reviewed by a second author to mitigate bias. The composition of the face, size of the product and contrast of the face with the background were easier to determine, although again were to an extent 
subjective to the authors interpretation of the images. The results illustrated in

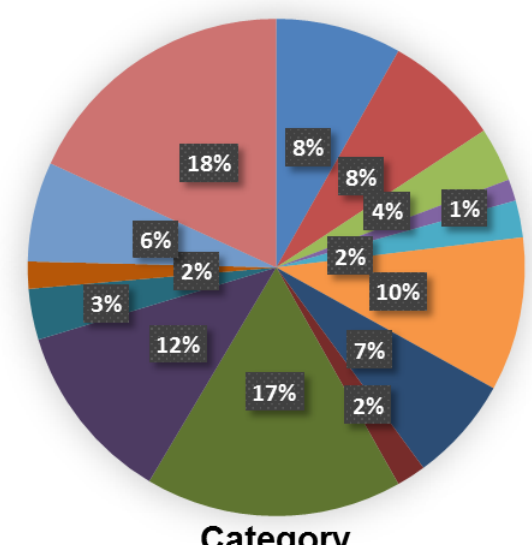

Category

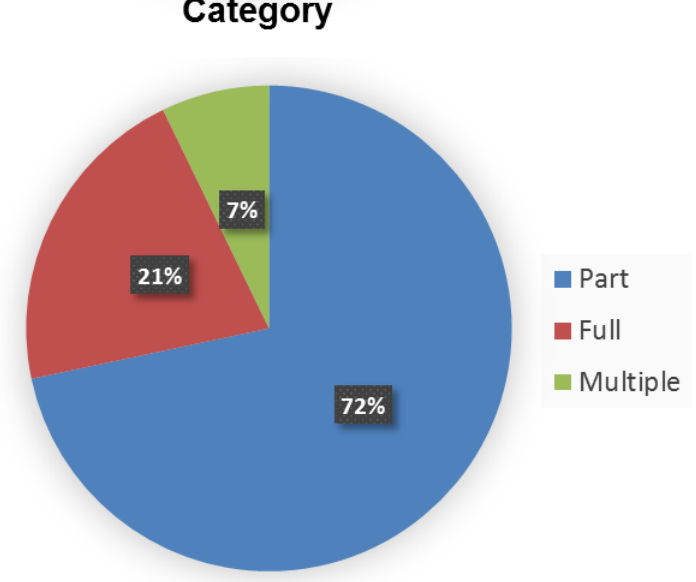

Composition
- Vehicle

- Kitchen

Bathroom

- Bedroom

- Garden

- Clothing

- Furniture

- Tools

- Gadgets

- Industrial

- Toy

- Graphics

- Packaging

- Component
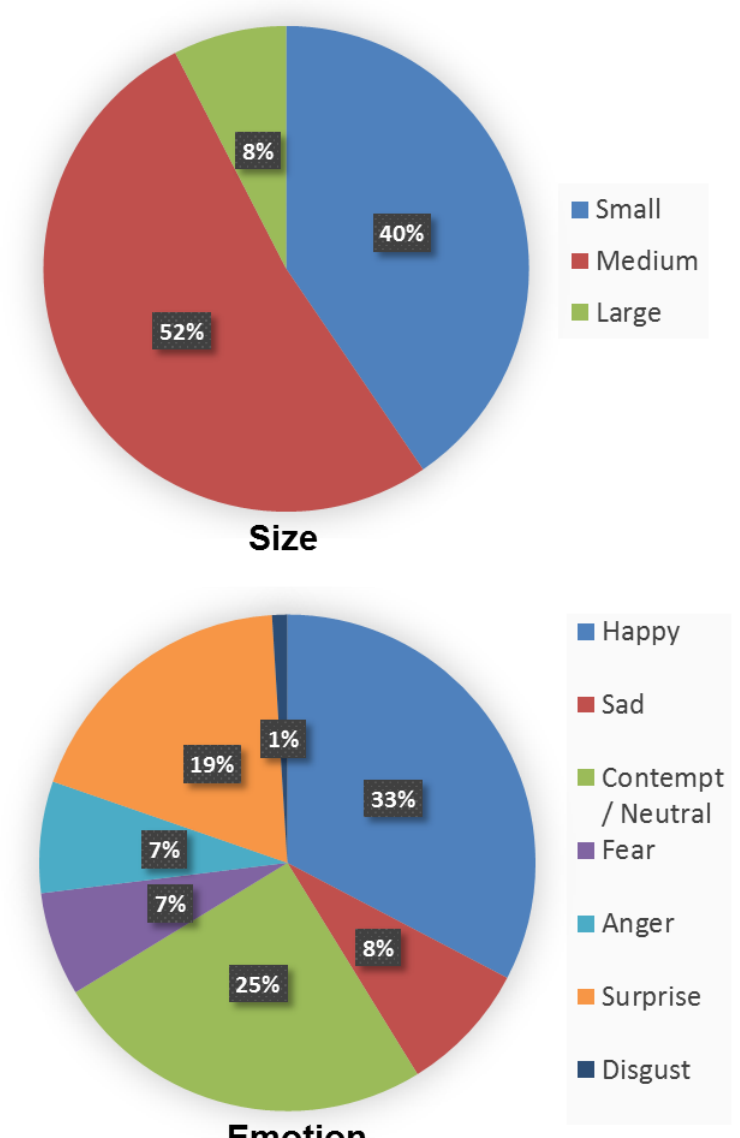

Figure 4 and discussed in Section 5 below. 


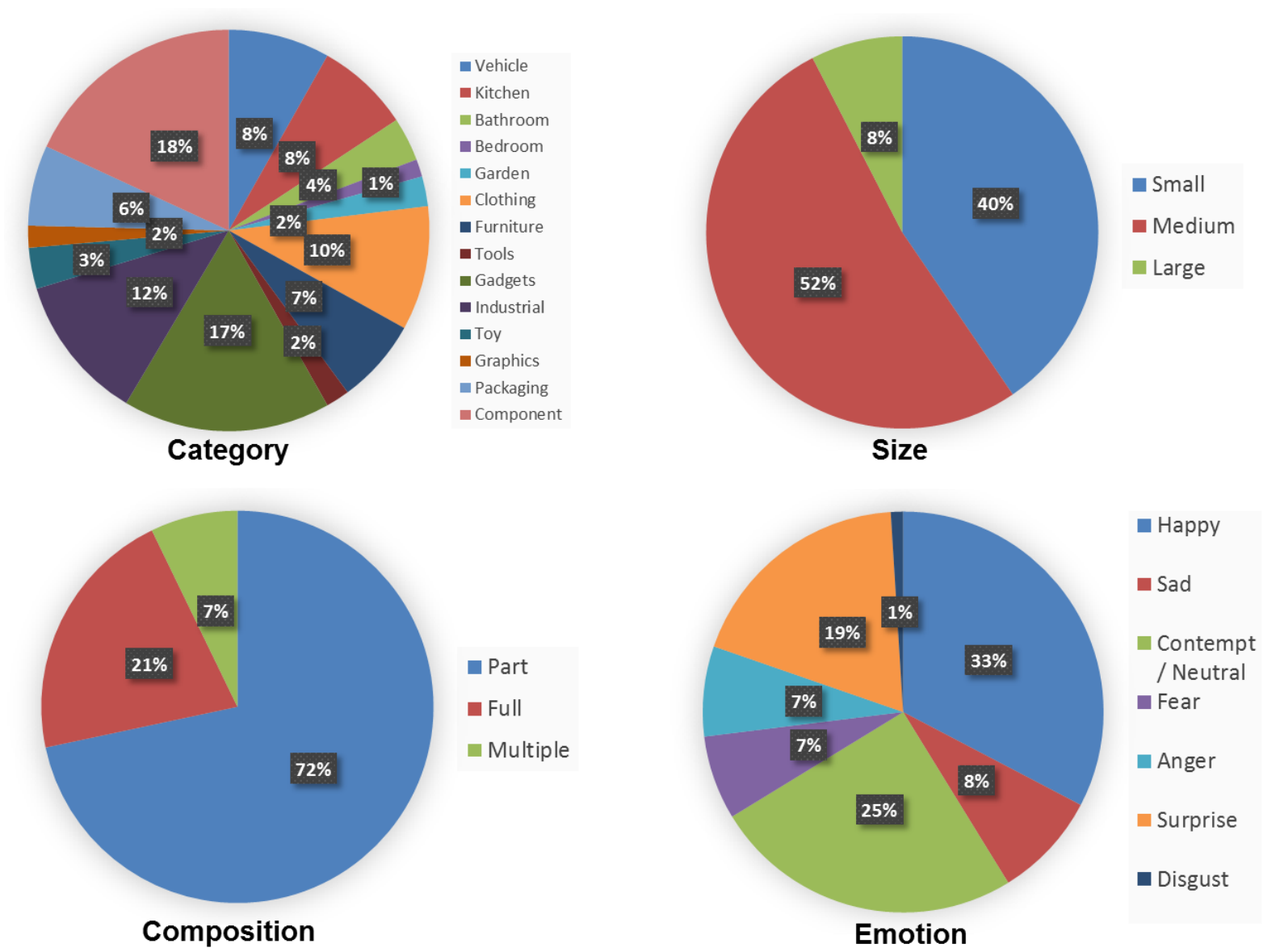

Figure 4: Images by product category, size, composition and emotion

\section{Discussion}

The initial finding that most images online were of products is perhaps surprising given the romantic ideals of seeing faces in clouds as children, or the fact that we are constantly navigating the built environment. But this possibly highlights that the products that we use and manipulate on a daily basis are more closely scrutinised than landscapes or buildings: when we hold, turn, and understand a product geometry we are more liable to reflect on its visual information. Also, the lines and symmetries afforded by modern manufacturing techniques lend themselves to faces emerging - many products have holes for screws, lines for slots or edges and other similar features.

\subsection{Prevalence}

In reviewing product categories, there was a fairly even spread across sectors but gadgets $(17 \%)$ and components $(18 \%)$ were the most common. In examining the database of images more closely, it was found that there were certain products that emerged repeatedly. These included (in no particular order): bags, card readers, cookers, intercoms, fire hydrants, bins, binoculars, bricks, boxes, and tooth brush holders. Some examples of these are shown in Figure 5. The regular geometries demanded by modern manufacturing processes, coupled with features like switches, dials, screw holes and so on means that there are many opportunities for faces to emerge. Many are dictated by product functionality in a similar fashion to the car elevation in particular, any time that symmetrical elements can be perceived as eyes then there is a high likelihood of product features being interpreted as a face. 


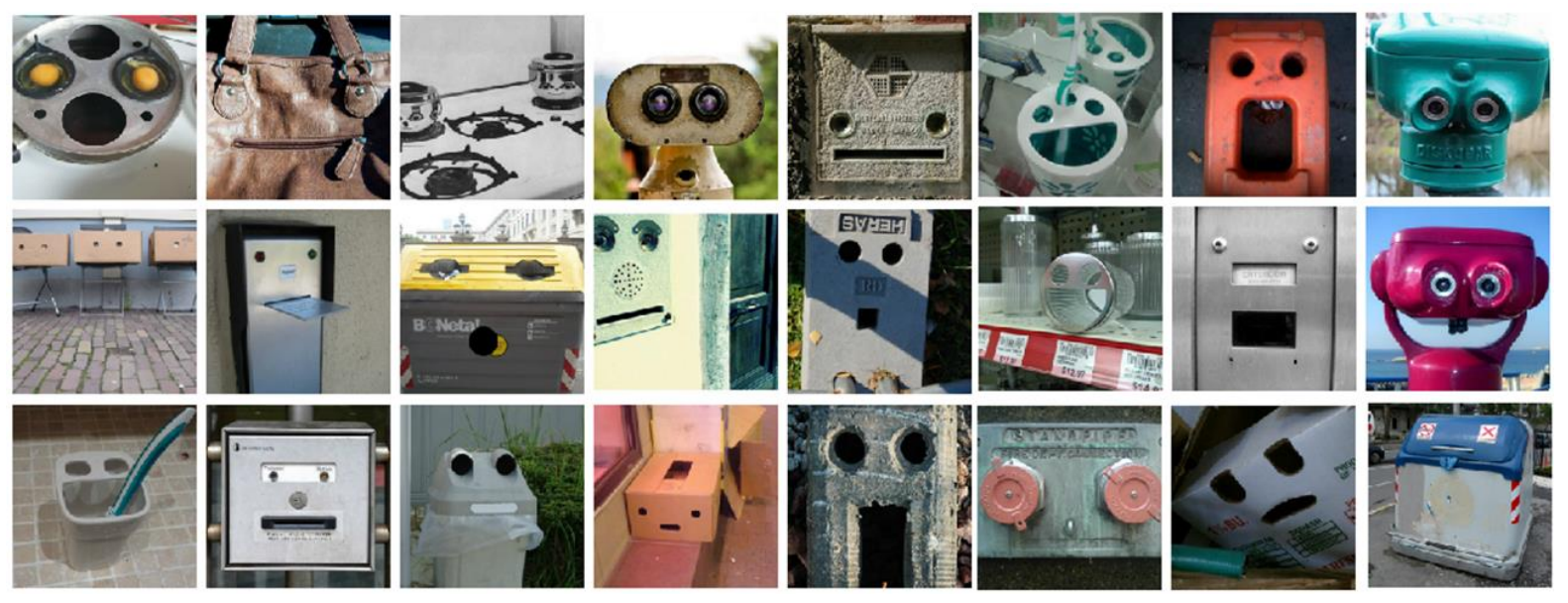

Figure 5: Examples of common or recurring products

\subsection{Size and composition}

The products in the images were then assessed as being small (handheld), medium (easily portable), or large (not easily portable). It was found that the majority of products were medium sized $(52 \%)$ or small $(40 \%)$, with only $8 \%$ of the images contained products that would be considered large. This suggests that faces were spotted in situations where people are interacting with products in the kind of proximity they would encounter a real face in conversation. When walking down the street, people do not often take the time to look up and around to spot patterns emerging from the larger objects in the build environment. When sitting at a desk, standing at a sink or operating a particular device the attention of the user is in greater proximity and more focussed, and therefore liable to find patterns of recognition (Figure 6(a)).

The faces identified in the products were identified as being a part of the product (for example the top of a bin), the whole product (a letterbox) or multiple products (bathroom fixtures viewed as one). It was found that a large majority (72\%) were a part of the product. It is often detailed design elements that exist on one side or sector of an object - the circular features that make holes, screws, bosses etc, and the linear features used for slots, ribs, runners etc. - that are arranged in such a way as to suggest eyes, noses and mouths. There were also a number of product faces that were unconventional in the geometry of internal composition: Figure 6(b) shows an industrial grabber that looks like an anthropomorphised cartoon snake.

For the viewer to successfully interpret this relies on cultural references, our level of familiarity with the product or technology, and conventions in terms of how animals are caricatured (in this case primarily through the eyes) to convey human qualities. This complex amalgam of influences touches on aspects of cultural design theories, such as the domestication of technology (Haddon 2007), and anthropological approaches to form characterisation (DiSalvo and Gemperle 2003). While it is beyond the scope of the work reported here, understanding the cultural framework to pareidolia is an intriguing aspect for further investigation.

\subsection{Emotional content}

The emotions assessed were happiness, sadness, fear, anger, surprise, disgust, and contempt. These were derived from Ekman's (Ekman 1980, 2001; Ekman et al. 1987) work on the universality of human expressions, and used recognised facial conventions to categorise the images, for example fear is expressed by eyebrows raised and wide mouth, anger by eyebrows 
down and closed mouth. Contempt is often conflated with a neutral face in Ekman's work and we therefore assigned faces with no discernible expression to this category. Most images (33\%) were interpreted as having happy expressions. Given the fact that a smiling face consists of tightened eyes, raised cheeks and diagonally upturned corners of the mouth, it seems unlikely that product geometries naturally lend themselves to this expression - more neutral lines and circles of neutral faces are typically the result of modern manufacturing processes and we may have therefore expected this category, which was second largest at $25 \%$, to have been greater. There may therefore be an element of self-congruence at play: previous studies have shown that consumers prefer products with a symbolic meaning that aligns with their own self-image (Govers and Schoormans 2005; Quester et al. 2000; Ross 1971). This indicates that we are actively looking for faces to be positive: if there is any suggestion of a wink or smile then this is more attuned to the playful "aesthetic aha!" that comes with pareidolia.

It is also in this category that deliberate anthropomorphism is likely to have been employed by the designer. In contrast, while sad faces were much less common $(8 \%)$ they were often the result of some kind of decay or change in environmental conditions, making them particularly evocative (Figure 6(c)). Disgust also rates highly (26\%), and while this is usually denoted by eyebrows down, wrinkled nose and mouth loose, it can be more subtly conveyed as contempt through largely neutral features with a crooked or asymmetrical mouth element. Often it was particular quirks in product geometry that led to one expression or another being conveyed. If the mouth was interpreted as a circular feature, this inevitably led to the emotion of surprise (19\%). Similarly, a straight or heavy feature across the brow invoked anger (7\%). And larger vertical distances between features often had the effect of suggesting fear (7\%). In our analysis, the use of Ekman's six emotions seemed ample to describe the range of emotions we encountered. This may be due to the fact that the faces are often made of simplistic geometry and lack the sophisticated structures to convey more advanced expressions such as serenity or apprehension. In intended pareidolia or anthropomorphism there is greater scope for designers to consciously replicate these by mimicking their subtleties in the product styling.

(a) size

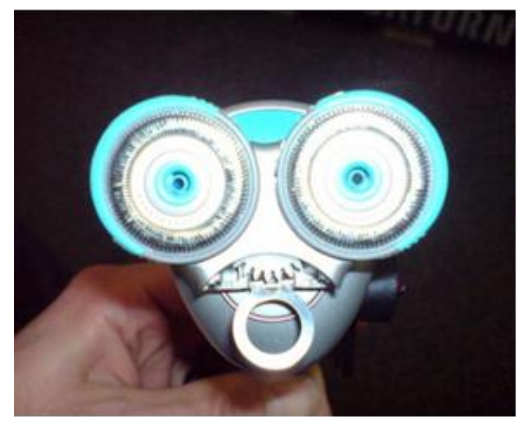

Faces were often found in human-sized or smaller surface areas in products that are manipulated or examined at conversational proximity. (b) composition

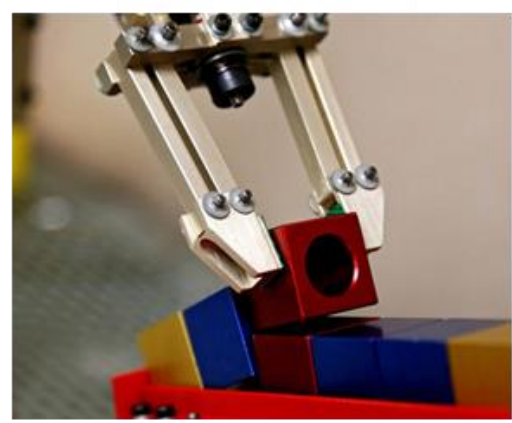

Faces usually emerged as a component, side or element of a product rather than a larger 'gestalt'. (c) emotion

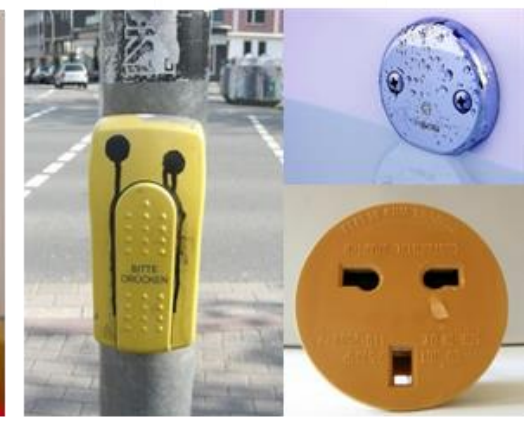

Sad faces often consisted of elements of decay or environmental change.

Figure 6: Examples of size, composition and emotion

\section{Conclusions}

This work set out to explore and document in more detail the phenomenon of pareidolia, and in the introduction we set out three main research questions:

- How prevalent is pareidolia in relation to product design? 
- What are the relevant product characteristics in instances where pareidolia occurs?

- What emotions are most commonly induced and how can these be harnessed by designers?

In addressing the prevalence of pareidolia, our review of literature on anthropomorphism and the use of faces in product design has shown that it is a compelling and increasingly acknowledged facet of how we interpret objects in the world around us. By surveying 2,309 images from across the internet, we found that products account for the majority $(62 \%)$ of instances. Given its ubiquity, this means it is critical for designers to consider pareidolia when constructing product configurations whether they wish their designs to manifest this phenomenon or not. Our second question considered the practical design implications in terms of product context and geometry construction. The prevalent characteristics we identified in products identified as having faces were size and composition. Medium-sized products that could be encountered as a normal 'conversation' or human-scale interaction accounted for the majority of cases. Additionally, it was usually the case that the face emerged in a facet of the product - a design detail, the face of one side or a grouping of features - rather than emerging from the gestalt form. Finally, we found that happy emotions were the most common in the interpretation of the product faces. Given that there is most scope for neutral faces (straight lines, circular holes) to emerge from modern manufacturing processes we suggest that there may be aspects of self-congruence and surprise at play in terms of user perception. These will vary depending on the state of mind of the user and context of the product.

The research in its current state has highlighted that this is an issue requiring consideration and provides a number of insights in terms of product characteristics and emotions. However, more work is required in order to develop tangible tools or methods that could be utilised by designers when configuring their designs and to this end we have identified two main directions for further work: more qualitative human-centred considerations and the determination of the geometrics and mechanics of faces. While we have touched upon issues regarding cultural interpretation and the role of self-congruence in finding and assigning emotion to emergent faces, we feel these would benefit from fuller consideration through the collection of detailed participant data. In terms of the composition of product faces, recognition and expression of emotion depends on certain geometric thresholds. Similarly, although we have reported on participant reaction to various geometries, we suggest abstracting and testing generic shapes and dimensions would be a fruitful investigation.

In summary, in the future we can anticipate more quantified guidelines on the use of pareidolia and anthropomorphism in design. We therefore hope that the results presented highlight the importance of the issue and that designers can review and consider the potential perception of users prior to finalising their detailed designs, whether it be their intention to utilise its effects or not.

\section{Acknowledgements}

Figure 2 lathe image credit: FreeImages.com/Jonathan Ogilvie. Figure 3 image credits (L to R): Wikicommons ID3346566; Wikicommons ID41971241; Freeimages.com/ Sasan Saidi; Wikicommons ID18968875; Yoshikazu Tsuno; Lobke Peers/ Shutterstock. All other images used courtesy of 'Faces in Places: A Photographic Collection of Faces found in Everyday Places' by Jody Smith, Ammonite Press, Lewes, UK. 


\section{References}

Aggarwal P, McGill Ann L (2007) Is That Car Smiling at Me? Schema Congruity as a Basis for Evaluating Anthropomorphized Products. Journal of Consumer Research 34 (4):468-479

Arnheim R (1969) Visual Thinking. University of California Press, Berkeley, CA

Baron-Cohen S, Wheelwright S, Hill J, Raste Y, Plumb I (2001) The "Reading the Mind in the Eyes" test revised version: A study with normal adults, and adults with Asperger syndrome or highfunctioning autism. Journal of child psychology and psychiatry 42 (2):241-251

Bartneck C, Kulić D, Croft E, Zoghbi S (2009) Measurement Instruments for the Anthropomorphism, Animacy, Likeability, Perceived Intelligence, and Perceived Safety of Robots. Int J of Soc Robotics 1 (1):71-81. doi:10.1007/s12369-008-0001-3

Canamero LD, Fredslund J How does it feel? emotional interaction with a humanoid lego robot. In: Proc. of American Association for Artificial Intelligence Fall Symposium, FS-00-04, 2000.

Carbon C-C (2011) The first 100 milliseconds of a face: on the microgenesis of early face processing. Perceptual and Motor Skills 113 (3):859-874. doi:10.2466/07.17.22.PMS.113.6.859-874

Carbon C-C, Grüter T, Grüter M, Weber JE, Lueschow A (2010) Dissociation of facial attractiveness and distinctiveness processing in congenital prosopagnosia. Visual Cognition 18 (5):641-654. doi:10.1080/13506280903462471

Chapman J (2005) Emotionally Durable Design: Objects, Experiences and Empathy. Earthscan, London, UK

Chitturi R, Raghunathan R, Mahajan V (2007) Form Versus Function:How the Intensities of Specific Emotions Evoked in Functional Versus Hedonic Trade-Offs Mediate Product Preferences. Journal of Marketing Research 44 (4):702-714. doi:doi:10.1509/jmkr.44.4.702

Claxton RP, Murray JB (1994) Object-subject interchangeability: A symbolic interactionist model of materialism. Advances in consumer research 21:422-422

Desmet P (2003) A multilayered model of product emotions. The Design Journal 6 (2):4-13

DiSalvo C, Gemperle F (2003) From seduction to fulfillment: the use of anthropomorphic form in design. Paper presented at the Proceedings of the 2003 international conference on Designing pleasurable products and interfaces, Pittsburgh, PA, USA,

Ekman P (1980) The face of man: Expressions of universal emotions in a New Guinea village. Garland STPM Press New York,

Ekman P (2001) Facial Expressions. In: Dalglend T, Power M (eds) Handbook of Cognition and Emotion. John Wiley \& Sons Ltd, Chichester, UK, pp 301-320

Ekman P, Friesen WV, O'Sullivan M, Chan A, Diacoyanni-Tarlatzis I, Heider K, Krause R, LeCompte WA, Pitcairn T, Ricci-Bitti PE, Scherer K, Tomita M, Tzavaras A (1987) Universals and cultural differences in the judgments of facial expressions of emotion. Journal of Personality and Social Psychology 53 (4):712-717. doi:10.1037/0022-3514.53.4.712

El Kaliouby R, Robinson P, Keates S Temporal context and the recognition of emotion from facial expression. In: Proceedings of HCI International Conference, 2003. Citeseer,

Epley N, Waytz A, Cacioppo JT (2007) On seeing human: A three-factor theory of anthropomorphism. Psychological Review 114 (4):864-886. doi:10.1037/0033-295X.114.4.864

Faerber SJ, Carbon C-C (2012) Jump on the innovator's train: cognitive principles for creating appreciation in innovative product designs. Research in Engineering Design:1-7. doi:10.1007/s00163-012-0148-7

Frazzetto G (2013) How We Feel: What Neuroscience Can - And Can't - Tell Us About Our Emotions. Transworld Publishers, London, UK

Govers PC, Hekkert P, Schoormans JP (2003) Happy, cute and tough: Can designers create a product personality that consumers understand. CRC Press, Boca Raton, FL,

Govers PC, Schoormans JP (2005) Product personality and its influence on consumer preference. Journal of Consumer Marketing 22 (4):189-197

Haddon L (2007) Roger Silverstone's legacies: domestication. New Media \& Society 9 (1):25-32

Hall S, Gay Pd (eds) (1996) Questions of Cultural Identity. SAGE Publications Ltd, London, England 
Haxby JV, Hoffman EA, Gobbini MI (2000) The distributed human neural system for face perception. Trends in cognitive sciences 4 (6):223-233

Hegel F, Spexard T, Wrede B, Horstmann G, Vogt T Playing a different imitation game: Interaction with an Empathic Android Robot. In: Humanoid Robots, 2006 6th IEEE-RAS International Conference on, 2006. IEEE, pp 56-61

Hoegg J, Alba JW (2011) Seeing Is Believing (Too Much): The Influence of Product Form on Perceptions of Functional Performance. Journal of Product Innovation Management 28 (3):346359. doi:10.1111/j.1540-5885.2011.00802.x

Landwehr JR, McGill AL, Herrmann A (2011) It's Got the Look: The Effect of Friendly and Aggressive "Facial" Expressions on Product Liking and Sales. Journal of Marketing 75 (3):132-146. doi:doi:10.1509/jmkg.75.3.132

Leder H, Carbon C-C (2005) When context hinders! Learn-test compatibility in face recognition. The Quarterly Journal of Experimental Psychology Section A 58 (2):235-250. doi:10.1080/02724980343000936

Lesher JH (2001) Xenophanes of Colophon: fragments, vol 4. University of Toronto Press,

Lo C-H, Chu C-H (2012) An investigation of the social-affective effects resulting from appearancerelated product models. Human Factors and Ergonomics in Manufacturing \& Service Industries:n/a-n/a. doi:10.1002/hfm.20352

McGilchrist I (2010) The Master and his Emissary: The Divided Brain and the Making of the Western World. Yale University Press, New Haven, CT

Mensvoort Kv, Grievink H-J (2011) Next Nature - Nature changes along with us.

Miesler L, Leder H, Herrmann A (2011) Isn't it cute: An evolutionary perspective of baby-schema effects in visual product designs. International Journal of Design 5 (3):17-30

Mitteroecker P, Gunz P, Windhager S, Schaefer K (2013) A brief review of shape, form, and allometry in geometric morphometrics, with applications to human facial morphology. Hystrix, the Italian Journal of Mammalogy 24 (1):59-66

Mori M (1970) The uncanny valley. Energy 7 (4):33-35

Morton J, Johnson MH (1991) CONSPEC and CONLERN: a two-process theory of infant face recognition. Psychological review 98 (2): 164

Mugge R, Govers PCM, Schoormans JPL (2009) The development and testing of a product personality scale. Design Studies 30 (3):287-302. doi:10.1016/j.destud.2008.10.002

Muth C, Carbon C-C (2013) The Aesthetic Aha: On the pleasure of having insights into Gestalt. Acta Psychologica 144 (1):25-30. doi:http://dx.doi.org/10.1016/j.actpsy.2013.05.001

Nam T-J, Kim C (2011) Design by Tangible Stories: Enriching Interactive Everyday Products with Ludic Value. International Journal of Design 5 (1)

Norman D (2004) Emotional Design: why we love (or hate) everyday things. Basic Books, New York

Ortony A, Turner TJ (1990) What's basic about basic emotions? Psychological review 97 (3):315

Purucker C, Sprott D, Herrmann A (2014) Consumer response to car fronts: eliciting biological preparedness with product design. Review of Managerial Science 8 (4):523-540. doi:10.1007/s11846-013-0116-2

Purucker C, Sprott DE, Herrmann A (2013) Consumer response to car fronts: eliciting biological preparedness with product design. Review of Managerial Science:1-18

Quester PG, Karunaratna A, Goh LK (2000) Self-congruity and product evaluation: a cross-cultural study. Journal of Consumer Marketing 17 (6):525-535

Ranscombe C, Hicks B, Mullineux G (2012) A method for exploring similarities and visual references to brand in the appearance of mature mass-market products. Design Studies 33 (5):496-520. doi:10.1016/j.destud.2012.04.001

Riek LD, Rabinowitch T-C, Chakrabarti B, Robinson P How anthropomorphism affects empathy toward robots. In: Proceedings of the 4th ACM/IEEE international conference on Human robot interaction, 2009. ACM, pp 245-246

Ross I (1971) Self-concept and brand preference. The Journal of Business 44 (1):38-50

Salem M, Eyssel F, Rohlfing K, Kopp S, Joublin F (2013) To err is human (-like): Effects of robot gesture on perceived anthropomorphism and likability. Int J of Soc Robotics 5 (3):313-323 
Sirgy MJ (1982) Self-concept in consumer behavior: a critical review. Journal of consumer research:287-300

Smith J (2010) Faces in Places: A Photographic Collection of Faces found in Everyday Places. Ammonite Press, Lewes, UK

Television Tropes and Idioms (2014) Sliding Scale of Anthropomorphism. http://tvtropes.org/pmwiki/pmwiki.php/Main/SlidingScaleOfAnthropomorphism. Accessed 15th March 2014

Ucko PJ (1962) The Interpretation of Prehistoric Anthropomorphic Figurines. The Journal of the Royal Anthropological Institute of Great Britain and Ireland 92 (1):38-54. doi:10.2307/2844320

Waytz A, Heafner J, Epley N (2014) The Mind in the Machine: Anthropomorphism Increases Trust in an Autonomous Vehicle. Journal of Experimental Social Psychology

Willis J, Todorov A (2006) First Impressions: Making Up Your Mind After a 100-Ms Exposure to a Face. Psychological Science 17 (7):592-598. doi:10.1111/j.1467-9280.2006.01750.x

Windhager S, Slice DE, Schaefer K, Oberzaucher E, Thorstensen T, Grammer K (2008) Face to face. Human Nature 19 (4):331-346

Winkielman P, Niedenthal PM, Oberman L (2008) The embodied emotional mind. In: Semin GR, Smith ER (eds) Embodied Grounding: Social, Cognitive, Affective, and Neuroscientific Approaches. Cambridge University Press, pp 263-288

Wu TY, Chang W (2007) The study of products with bios forms in conveying pleasure. Paper presented at the International Association of Societies of Design Research, Hong Kong, China, 12-15 November 